УДК 332.144

И. А. Хасаншин

ФГОБУ ВО «Поволжский государственный университет телекоммуникаций и информатики», Самара, e-mail: ildar8000@mail.ru

Ю. А. Токарев

ФГОУ ВО «Самарский государственный экономический университет», Самара, e-mail: tokarev_ya@mail.ru

\title{
ПРОГНОЗ РАЗВИТИЯ ИНФОРМАЦИОННОЙ ИНФРАСТРУКТУРЫ ПРЕДПРИНИМАТЕЛЬСКОЙ ДЕЯТЕЛЬНОСТИ НА УРОВНЕ РЕГИОНА
}

Ключевые слова: предпринимательство, инфраструктура, предпринимательская активность, прогноз.

Согласно Указу Президента РФ «О национальных целях и стратегических задачах развития Российской Федерации на период до 2024 года» (№ 204 от 7 мая 2018 г.), Правительству России необходимо достичь следующую стратегическую цель: создать «устойчивую и безопасную информационно-телекоммуникационную инфраструктуру высокоскоростной передачи, обработки и хранения больших объемов данных, доступной для всех организаций». Это есть важный «кирпичик» в фундаменте информационной инфраструктуры предпринимательской деятельности. Мы можем выделить большое число параметров, оказывающих прямое (стимулирующее) или обратное воздействие на показатели инфраструктурного обеспечения предпринимательской деятельности. Познавательный аспект состоит в выборе конкретных показателей (индикаторов). Учитывая специфику нашего исследования, мы выдвигаем требование к этим показателям. Они должны представлять показатели технической оснащённости и обеспеченности предприятий информационно-коммуникационными технологиями. Отметим, что официальная статистика предоставляет ограниченное число информации подобного вида. Более того, данные не всегда сопоставимы за длительный временной период. В нашем исследовании мы в основном оперируем данными Росстата за 2003-2017 годы. Это достаточно длинный временной ряд, что позволит нам в итоге сделать прогноз на среднесрочный период.

\section{Введение}

Развитие системы инфраструктурного обеспечения предпринимательской деятельности в экономике тесно связано с уровнем развития ИКТ. В свою очередь, состояние инфраструктуры должно коррелировать с предпринимательской активностью предприятий, которая гипотетически выступает драйвером роста объёма инновационных товаров и услуг в экономике.

В условиях рыночной экономики коренным образом меняется подход к управлению: от функционального к бизнес-ориентированному; кардинально меняется и роль информационных технологий. Ориентация на управление на основе бизнес-процессов обеспечивает конкурентное преимущество для организации в условиях острейшей конкуренции, а управление на основе бизнес-процессов не может эффективно реализовываться без применения информационных технологий и систем.

Согласно Указу Президента РФ «О национальных целях и стратегических задачах развития Российской Фе- дерации на период до 2024 года» (№ 204 от 7 мая 2018 г.), Правительству России необходимо достичь следующую стратегическую цель: создать «устойчивую и безопасную информационно-телекоммуникационную инфраструктуру высокоскоростной передачи, обработки и хранения больших объемов данных, доступной для всех организаций». Это есть важный «кирпичик» в фундаменте информационной инфраструктуры предпринимательской деятельности.

Цель исследования. Мы можем выделить большое число параметров, оказывающих прямое (стимулирующее) или обратное воздействие на показатели инфраструктурного обеспечения предпринимательской деятельности. Познавательный аспект состоит в выборе конкретных показателей (индикаторов). Учитывая специфику нашего исследования, мы выдвигаем требование к этим показателям. Они должны представлять показатели технической оснащённости и обеспеченности предприятий информационнокоммуникационными технологиями. 


\section{Материалы и методы исследования}

Отметим, что официальная статистика предоставляет ограниченное число информации подобного вида. Более того, данные не всегда сопоставимы за длительный временной период. В нашем исследовании мы в основном оперируем данными Росстата за 2003-2017 годы. Это достаточно длинный временной ряд, что позволит нам в итоге сделать прогноз на среднесрочный период.

В нашем исследовании показателями информационной инфраструктуры предпринимательской деятельности являются:

$X_{1}$ - удельный вес организаций, использовавших Интернет (процент от всех обследованных организаций);

$X_{2}$ - удельный вес организаций, имевших веб-сайт в сети Интернет (процент от всех обследованных организаций);

$X_{3}$ - удельный вес организаций, использовавших персональные компьютеры (ПК) (процент от всех обследованных организаций);

$X_{4}$ - число персональных компьютеров на 100 работников - всего, шт.;

$X_{5}$ - затраты на ИКТ (млрд руб.).

Временной ряд представляет собой последовательность данных, описывающих объект в последовательные моменты времени. В отличие от анализа случайных выборок, анализ временных рядов основывается на предположении, что последовательные данные наблюда- ются через равные промежутки времени. Рассмотрим изменение во времени показателей $X_{1} \ldots X_{4}$ (табл. 1). Тенденции, характерные для страны в целом, присущи и большинству регионов.

Таблица 1 наглядно показывает увеличение обеспеченности персонала предприятий компьютерной техникой, что влечёт за собой применение специальных программных средств (антивирусных программ, электронных справочноправовых систем и т.д.), использование глобальных информационных систем (Интернет, Экстранет, Интранет), ведение электронного документооборота.

В то же время заметим, что доступ к сети Интернет имеет лишь 2/3 компьютеров (в 2017 году - 66\%). Во многом это связано с тем, что значительная часть ЭВМ подключена к корпоративным или ведомственным сетям и не требует (по разным причинам) выхода в общедоступную сеть. Заметим, что по данному показателю 50-процентный рубеж оказался достигнут только в 2010 году.

Использование интернета в организациях проводится достаточно активно. Уже в течение 5 лет доля организаций, использовавших Интернет, варьируется около отметки 88-89\%. Тем удивительнее тот факт, что до сих пор более половины организаций не имеют свой вебсайт. Наиболее низка их доля в сфере малого бизнеса, связанного с продажей

Таблица 1

Динамика показателей информационной инфраструктуры предпринимательской деятельности в Российской Федерации

\begin{tabular}{|c|c|c|c|c|c|}
\hline Годы & $X_{1}$ & $X_{2}$ & $X_{3}$ & $X_{4}$ & $X_{5}$ \\
\hline 2003 & 43,4 & 13,5 & 84,6 & 18 & 164,6 \\
\hline 2004 & 48,8 & 14,4 & 87,6 & 20 & 168,4 \\
\hline 2005 & 53,3 & 14,8 & 91,1 & 23 & 215,3 \\
\hline 2006 & 61,3 & 21,1 & 93,3 & 26 & 252,0 \\
\hline 2007 & 67,8 & 19,8 & 93,3 & 29 & 299,4 \\
\hline 2008 & 73,7 & 22,8 & 93,7 & 32 & 372,7 \\
\hline 2009 & 78,3 & 24,1 & 93,7 & 35 & 421,4 \\
\hline 2010 & 82,4 & 28,5 & 93,8 & 36 & 515,6 \\
\hline 2011 & 84,8 & 33,0 & 94,1 & 39 & 603,0 \\
\hline 2012 & 86,9 & 37,8 & 94,0 & 43 & 842,7 \\
\hline 2013 & 88,1 & 41,3 & 94,0 & 44 & 1245,7 \\
\hline 2014 & 89,0 & 40,3 & 93,8 & 47 & 1174,9 \\
\hline 2015 & 88,1 & 42,6 & 92,3 & 49 & 1153,1 \\
\hline 2016 & 88,7 & 45,9 & 92,4 & 49 & 1249,2 \\
\hline 2017 & 88,9 & 47,4 & 92,1 & 50 & 1487,6 \\
\hline
\end{tabular}


продовольственных товаров, швейными услугами, ремонтом обуви и т. п. (доля таких организаций с собственным интернет-сайтом около $20 \%$ ). Наоборот, в создании и продвижении своих веб-сайтов заинтересованы туристские, страховые, банковские организации, крупные торговые сети, букмекерские конторы и т. Д.

\section{Результаты исследования и их обсуждение}

На рис. 1 мы наглядно покажем относительное изменение рассмотренных выше показателей. Мы не включим в расчёты только показатель «Затраты на ИКТ», так как в силу своего стоимостного характера он подвержен влиянию инфляционного фактора.

Мы рассматриваем показатели информационной инфраструктуры не как обособленные, а как факторные индикаторы, влияющие на инновационный аспект предпринимательской деятельности. Соответственно, возникает задача выделить показатели, которые дают количественную оценку этой деятельности:

$Y_{1}$ - инновационная активность организаций (удельный вес организаций, осушествлявших технологические, организационные, маркетинговые инновации в отчетном году, в общем числе обследованных организаций), \%;

$Y_{2}$ - удельный вес инновационных товаров, работ, услуг в общем объеме отгруженных товаров, выполненных работ, услуг (\%).

Чётким критерием отбора факторов является парный коэффициент корреляции $r_{x y}$, измеряющий тесноту взаимосвязи с каждым результативным признаком. Его значения меняются в интервале $|0 \ldots 1|$. Чем ближе значение к $|1|$, тем теснее взаимосвязь. Она считается существенной при значении $r_{x y} \geq|0,5|$. Это число и послужило критерием отбора факторов.

Значения парных коэффициентов корреляции приведены в табл. 2.

Таблица 2

Парные коэффициенты корреляции

\begin{tabular}{|c|c|c|}
\hline & $Y_{1}$ & $Y_{2}$ \\
\hline$X_{1}$ & $-0,126$ & 0,702 \\
\hline$X_{2}$ & $-0,296$ & 0,861 \\
\hline$X_{3}$ & 0,208 & 0,342 \\
\hline$X_{4}$ & $-0,277$ & 0,811 \\
\hline$X_{5}$ & $-0,359$ & 0,879 \\
\hline
\end{tabular}

Таким образом, табл. 2 говорит нам о том, что показатели информационной инфраструктуры предпринимательской деятельности имеют заметную корреляцию с инновационными индикаторами, однако она носит разнонаправленный и неравномерный характер.

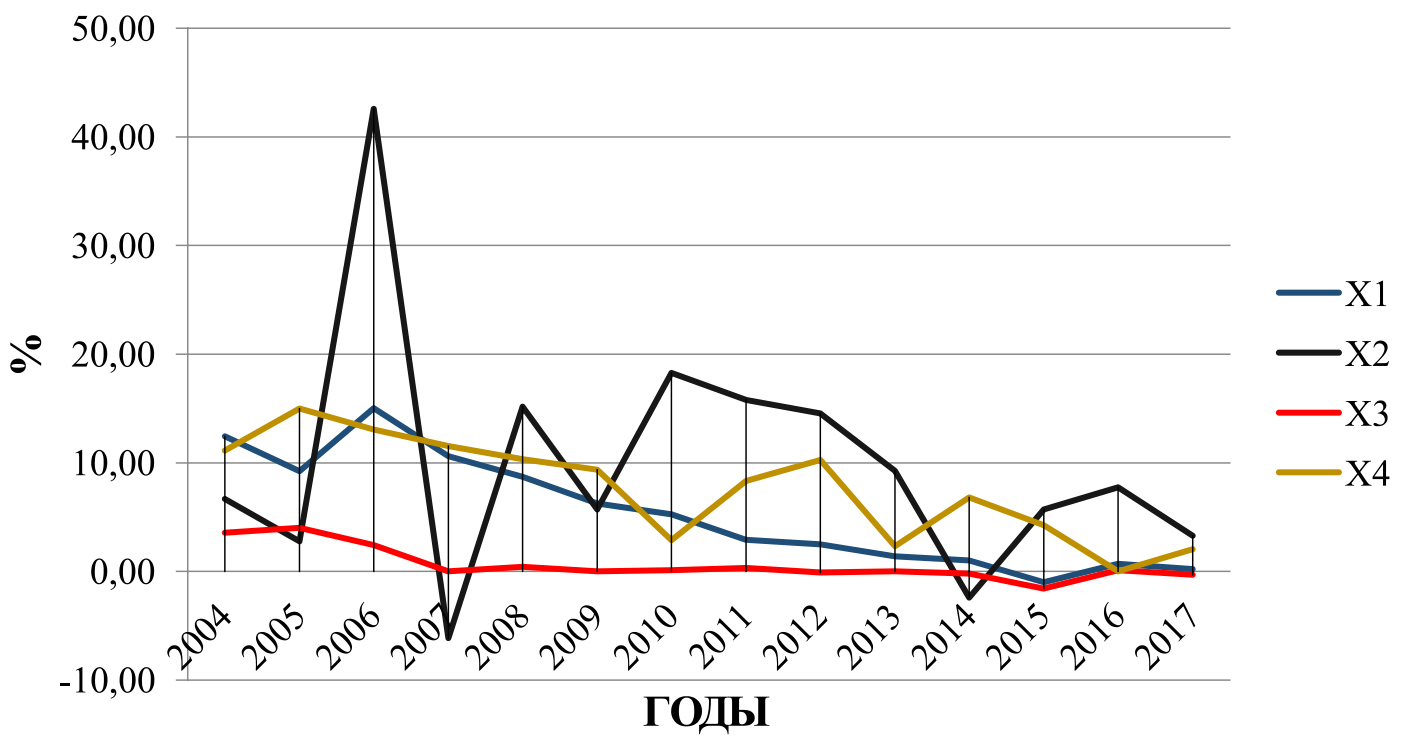

Рис. 1. Цепные прирость (\%) показателей информаџионной инфраструктуры предпринимательской деятельности в Российской Федерации 
Так, для инновационной активности предпринимательской деятельности организаций наличие компонентов информационной инфраструктуры не носит решающего характера и часто становится тормозом её развития (например, рост затрат на ИКТ в регионах имеет обратную связь с инновационной активностью предпринимателей).

Для показателя производства инновационной продукции, наоборот, необходима соответствующая информационная инфраструктура предпринимательской деятельности. Важными факторами является широкая обеспеченность компьютерной техникой работников на предприятиях $\left(r_{y 2 \times 4}=0,811\right)$, наличие интернет-сайта $(r \stackrel{y 2 x 4}{\geq 0,861)})$ и высокие затраты на ИКТ $\left(\dot{r}_{y 2 x 5} \geq 0,879\right)$.

Одна из основных задач исследования состоит в изучении причинноследственных связей, в количественной оценке роли каждого фактора, влияющего на предпринимательскую деятельность в экономике. Классическим, традиционным, общепринятым методом решения этих задач в экономических исследованиях является корреляционнорегрессионный анализ (КРА).

Линейное уравнение регрессии (линейная регрессионная модель) будет иметь вид:

$$
Y=b_{0}+b_{1} X_{1}+b_{2} X_{2}+\ldots+b_{n} X_{n}+\xi,
$$

где $b_{0}$ и $b_{1 . n}$ - параметры уравнения регрессии $\left(b_{1, n}^{n}\right.$ - коэффициенты регрессии); $Y$ - зависимая (объясняемая, результативная) переменная; $X_{i}-$ независимая (объясняющая, факторная) переменная; $\xi$ - ошибка (случайная компонента).

Коэффициент регрессии $b_{i}$ показывает, на сколько единиц в среднем меняется значение результативного признака $Y$ при изменении признака $X_{i}$ на 1 ед.

Для практического использования регрессионных моделей большое значение имеет их адекватность, то есть соответствие фактическим статистическим данным.

В экономике принята устоявшаяся схема проверки адекватности построенных моделей, которая проводится по следующим направлениям:

- проверка статистической значимости коэффициентов регрессии (t-критерий Стьюдента);
- проверка общего качества (т. е. значимости) уравнения регрессии в целом (F-критерий Фишера).

Проверить значимость (качество) уравнения регрессии - значит, установить, соответствует ли математическая модель, выражающая зависимость между переменными, экспериментальным данным, достаточно ли включенных в уравнение объясняющих переменных для описания зависимой переменной. Если модель не адекватна, то это говорит, прежде всего, об отсутствии значимой взаимосвязи между факторами и результатом.

При анализе адекватности уравнения регрессии (модели) исследуемому процессу, возможны следующие варианты.

1. Построенная модель на основе $F$-критерия Фишера в целом адекватна и все коэффициенты регрессии значимы по $t$-критерию. Такая модель может быть использована для принятия решений и осуществления прогнозов.

2 . Модель по $F$-критерию Фишер адекватна, но часть коэффициентов не значима. Модель пригодна для принятия некоторых решений, но не для прогнозов.

3 . Модель по $F$-критерию неадекватна, все коэффициенты регрессии не значимы. Модель полностью считается неадекватной. На ее основе не принимаются решения и не осуществляются прогнозы.

В итоге нами построено две регрессионных модели, математически отражающих взаимосвязи по результативному показателю с наиболее важными факторами.

Модель по показателю удельного веса инновационных товаров, работ, услуг $\left(Y_{2}\right)$ имеет следующий вид:

$$
\widehat{Y}_{2}=4,32+0,003 x_{5},
$$

где $X_{5}$ - затраты на ИКТ (млрд руб.).

Количественно взаимосвязь признаков измеряют коэффициенты корреляции и детерминации. Для качества построенной модели имеет особое значение второй из них. Коэффициент детерминации $R^{2}$ принимает значения в диапазоне от нуля до единицы. Он показывает, какая часть вариации результативного признака $(Y)$ объяснена уравнением регрессии. Чем больше $R^{2}$, тем 
большая часть вариации результативного признака объясняется уравнением регрессии и тем лучше уравнение регрессии описывает исходные данные. При отсутствии зависимости между $Y$ и $X$ коэффициент детерминации будет близок к 0. Таким образом, коэффициент детерминации $R^{2}$ также может применяться для оценки качества (точности) уравнения регрессии.

Данная модель имеет высокие качественные показатели (коэффициент множественной корреляции равен 0,879 , коэффициент детерминации 0,772 , модель значима по $F$-критерию Фишера).

При увеличении затрат на ИКТ на 1 млрд руб. доля инновационных товаров возрастает в среднем на 0,003 процентных пункта. Именно предприятия с большими затратами на ИКТ являются наиболее заинтересованными в развитии инноваций.

Рассмотрим модель с другим ключевым фактором:

$$
\hat{Y}=2,88+0,120 x_{2},
$$

где $X_{2}-$ удельный вес организаций, имевших веб-сайт в сети Интернет (\%).

Bсе коэффициенты регрессии значимы по ш-критерию на 5-процентом уровне достоверности. Поясним экономический смысл. Рост доли организаций, имевших веб-сайт, на 1 процентный пункт, приводит к увеличению доли инновационной продукции в среднем на 0,12 процентных пункта, следовательно, есть прямая зависимость между активностью в интернет-сфере и результативностью инновационной предпринимательской деятельности.

Значение коэффициента детерминации в нашей модели $(0,741)$ показывает, что вариация инновационной активности организаций на 74,1 \% определяется вариацией удельного веса организаций, имеющих сайт, а на 25,6\% - вариацией прочих факторов.

Коэффициент корреляции имеет значение в данной модели 0,861 - это весьма высокий показатель.

Модель по показателю $Y_{1}$ - «Инновационная активность организаций»нами не была построена по причине её статистической незначимости (низкие значения F-критерия и t-критерия).
Рассмотрим динамику удельного веса инновационных товаров, работ, услуг в общем объеме отгруженных товаров, выполненных работ, услуг в Российской Федерации и в Приволжском федеральном округе ПФО (табл. 3). Этот временной ряд более длинный (в отличие от табл. 1), так как имеется соответствующая статистическая информация.

\section{Таблица 3}

Динамика удельного веса инновационных товаров, работ, услуг (\%) в Российской Федерации и Приволжском федеральном округе

\begin{tabular}{|c|c|c|}
\hline Годы & РФ & ПФО \\
\hline 2000 & 4,4 & 6,2 \\
\hline 2001 & 4,1 & 7,2 \\
\hline 2002 & 4,2 & 4,9 \\
\hline 2003 & 4,6 & 5,9 \\
\hline 2004 & 5,3 & 8,8 \\
\hline 2005 & 5,0 & 10,5 \\
\hline 2006 & 4,7 & 11,3 \\
\hline 2007 & 4,6 & 10,6 \\
\hline 2008 & 5,0 & 9,8 \\
\hline 2009 & 4,5 & 9,3 \\
\hline 2010 & 4,8 & 10,2 \\
\hline 2011 & 6,3 & 11,3 \\
\hline 2012 & 8,0 & 12,7 \\
\hline 2013 & 9,2 & 14,2 \\
\hline 2014 & 8,7 & 13,8 \\
\hline 2015 & 8,4 & 13,0 \\
\hline 2016 & 8,5 & 14,1 \\
\hline 2017 & 7,2 & 13,3 \\
\hline
\end{tabular}

Отметим, что Приволжский округ в настоящее время лидирует в стране по данному показателю, существенно опережая другие федеральные округа (для сравнения - на втором месте находится Южный ФО со значением 9,0\%, а в других округах доля ниже 9\%).

На рис. 2 показаны значения цепных приростов по России и ПФО.

Графики динамики по обеим территориям синхронизировались только после 2012 года. В целом, динамика удельного веса инновационной продукции по России меньше подвержена колебаниям. Это можно объяснить тем, что данный показатель фактически «растворяет» в себе все частные значения по субъектам Федерации и нивелирует их большие и маленькие отклонения от среднего уровня. 


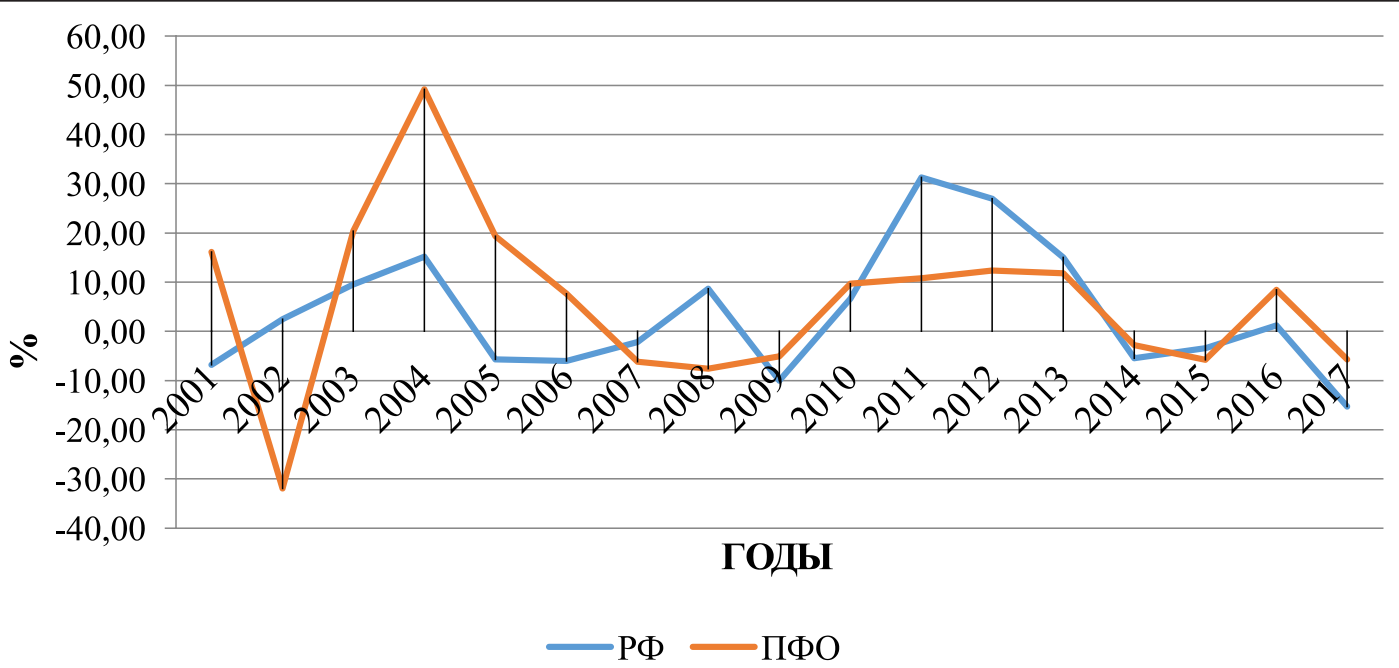

Рис. 2. Цепные приросты (\%) удельного веса инноващионных товаров, работ, услуг (по РФ и ПФО)

Пик предпринимательской активности российских организаций пришёлся на 2011-2012 годы, когда в среднем каждое десятое предприятие вкладывало средства в инновации разного вида. В Приволжском федеральном округе эта стадия была на несколько лет раньше если в 2002 году удельный вес инновационной продукции составил весьма низкие $4,9 \%$, то уже в 2006 году он достиг значения $11,3 \%$. Этим и объясняются высокие значения цепных приростов в данный период.

В целом в 2014-2015 гг. в силу действия антироссийских санкций деловая активность в предпринимательской сфере немного уменьшилась. Однако общий тренд имеет положительный вектор. Более того, в 2016 году цепные приросты снова превысили нулевую отметку.

Для выражения основной тенденции развития явления применяется аналитическое выравнивание. Оно связано с построением трендовых моделей и предусматривает возможность прогнозирования на их основе.

При этом основная тенденция рассматривается как функция времени (кривая роста): $Y=f(t)$. Она может быть выражена различными видами уравнений: линейными, параболическими и т.д.

Функция подбирается как на основе визуального анализа графического изображения ряда, так и с помощью специальных критериев (показателей).

Динамику доли удельного веса инновационных товаров, работ, услуг в России можно математически представить уравнением тренда в виде полиномиальной функции второго порядка (рис. 3):

$$
\widehat{Y}_{2}=4,07+0,013 t+0,042 t^{2} .
$$

Качество этой модели подтверждается высоким значением коэффициента детерминации 0,737. Таким образом, $73,7 \%$ изменений удельного веса инновационных товаров объясняется фактором времени.

На основе данной модели мы выполнили точечный прогноз на 20202025 годы по стране в целом. Прогнозные значения составят: в 2020 году $9,51 \%$, в 2021 году - 10,06\%, в 2022 году $-10,63 \%$, в 2023 году $11,23 \%$, в 2024 году - $12,50 \%$, в 2025 году - $13,17 \%$.

Прогноз носит позитивный характер, несмотря на некоторый спад относительного объёма инновационных товаров и услуг в экономике страны. Важным моментом в настоящее время является повышенное внимание государства к инновационному развитию предпринимательства, что отражено в соответствующих программных и стратегических доку ментах федерального урвоня (например, национальная программа «Цифровая экономика Российской Федерации»).

На рис. 4 показана динамика аналогичного показателя по Приволжскому федеральному округу. 


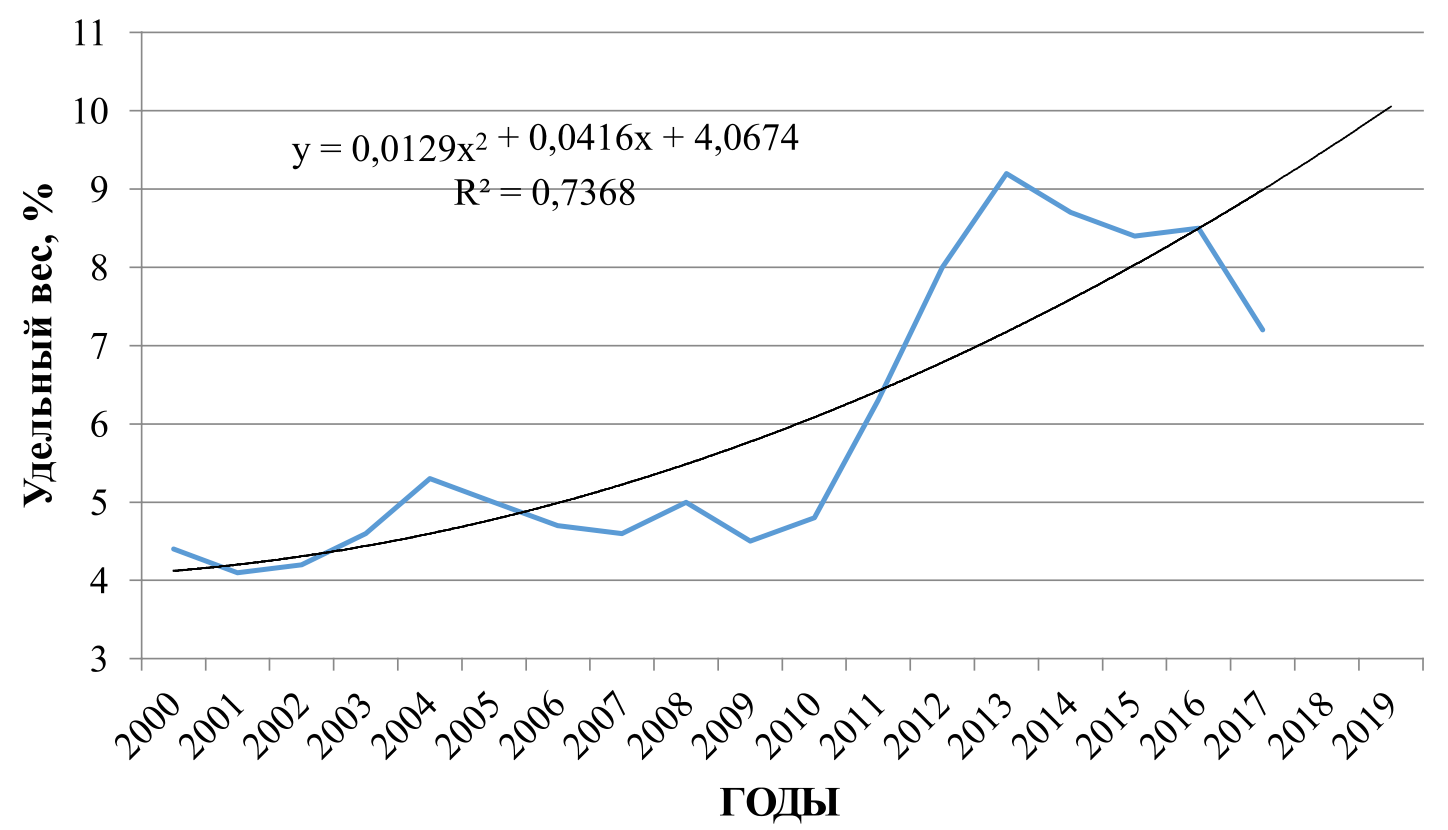

Рис. 3. Трендовая модель удельного веса инноващионньх товаров, работ, услуг (по РФ), \%

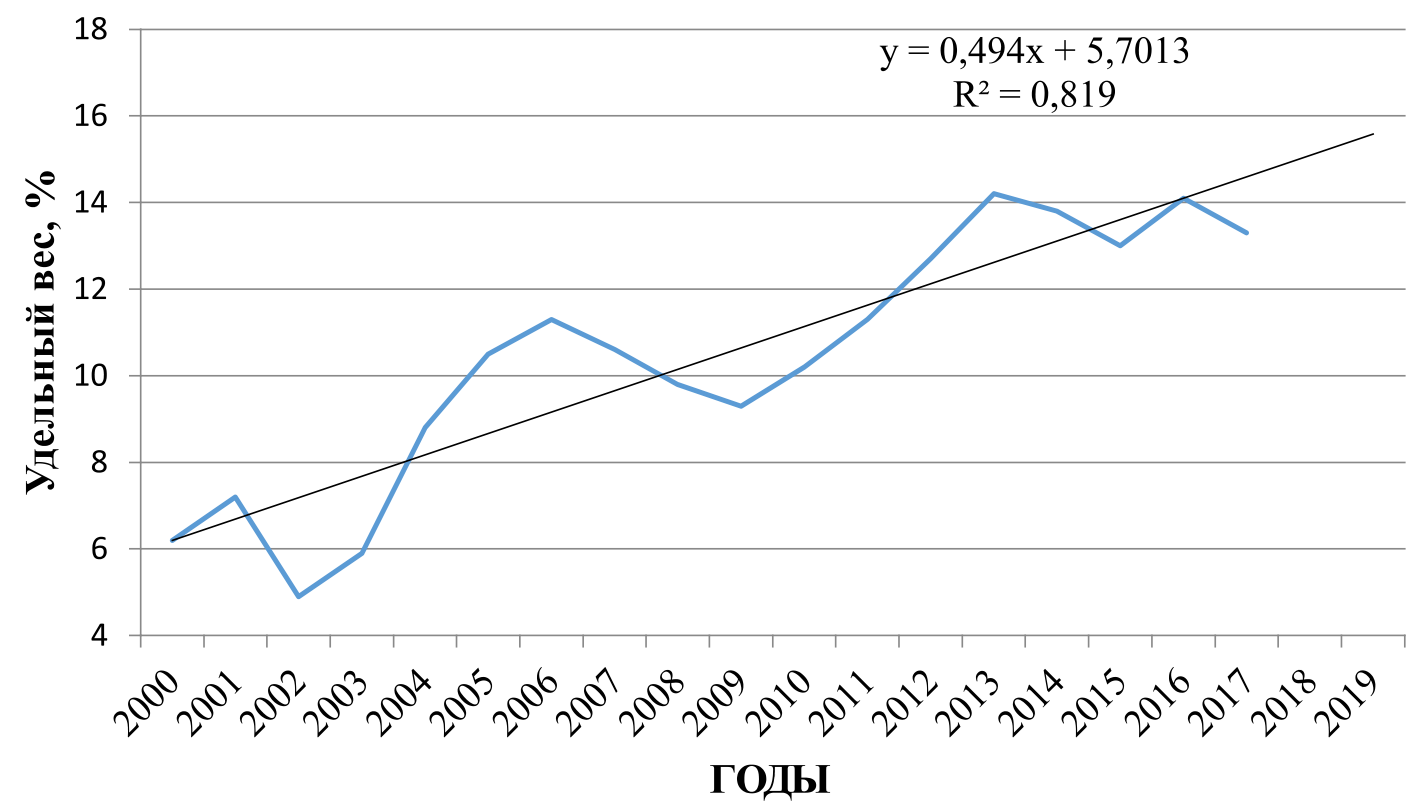

Рис. 4. Трендовая модель удельного веса инновачионных товаров, работ, услуг (по ПФО), \%

Тенденция удельного веса производства инновационных товаров (работ, услуг) хорошо аппроксимируется линейным трендом. Уравнение тренда имеет вид:

$$
\widehat{Y}_{2}=5,70+0,49 t \text {. }
$$

Мы видим тенденцию к росту рассматриваемого показателя (теоретически доля инновационной продукции должна возрастать ежегодно в среднем на 0,49 про- центных пунктов). Прогноз на 2020 год $15,58 \%$, на 2021 год - 16,08\%, на 2022 год$16,57 \%$, на 2023 год - 17,06\%, на 2024 год$17,56 \%$, на 2025 год $-18,05 \%$. Линейная трендовая модель на 81,9\% аппроксимирует эмпирические уровни временного ряда.

Применение полиномиальных трендовых моделей более высоких порядков может быть оправдано математически (в силу больших значений коэффициента аппроксимации), однако не имеет 
экономического смысла - прогноз по данным моделям показывает значительное сокращение инновационной продукции в ближайшие годы, что не согласуется с общим положительным трендом последних лет и курсом федерального Правительства на создание инновационной экономики.

Отметим основные сложности, которые могут возникнуть в развитии информационной инфраструктуры инновационной деятельности региона.

Несмотря на то, что развитие высокотехнологичного производства необходимо для каждой страны, в регионах России оно встречает на своем пути определенные трудности. В нашей стране есть ряд корпораций-гигантов, единственных в своем роде, которые занимаются выпуском высокотехнологичной продукции.

Выход на рынок небольших компаний в этих условиях представляется проблематичным. Дело здесь не только в большой конкуренции на рынке, но, прежде всего в налоговой ставке, непосильной для зарождающейся компании и отсутствии инвестиций. Курс государства на ускорение инновационного развития взят уже несколько лет назад, однако подъем высокотехнологичного производства идет крайне медленно, хотя есть много идей, открытий и разработок, готовых конкурировать с зарубежными аналогами. Необходимо помнить, что совокупность всех высокотехнологических областей играет важную роль в развитии экономики страны, т. к. на высокотехнологичных предприятиях разрабатываются и внедряются технологии, которые могут быть использованы в менее технологичных областях, повышая конкурентоспособность продукции, выпускаемой такими предприятиями.

Для решения проблемы технологического отставания России от ведущих развитых стран мира, необходимо создать адекватные механизмы инвестирования, выявить и устранить препятствия на пути продвижения технологий на рынок, т. е. создать благоприятную среду для возникновения и развития инноваций.

Согласно «Стратегии инновационного развития РФ на период до 2020 года»
[1], в целях экономического развития России приоритетное значение играет «роль переориентации экономики страны с «ресурсного» менеджмента, который основан на эксплуатации материальных и иных ресурсов, на инновационный тип управления, который базируется на непрерывном процессе разработки новшеств и коммерциализации и инноваций и невозможный без формирования конкурентоспособной национальной инновационной системы» [2].

Анализируя мнение ведущих учёных, специалистов в области прогнозирования предпринимательской активности, мы делаем вывод о том, что из вышесказанного вытекает необходимость усиления мер государственного регулирования предпринимательской деятельности региона. Так, Удальцова Н.Л. предлагает повысить спрос на инновации со стороны частного сектора, то есть расширить число инновационно-активных отраслей экономики [3]. Абрамова К.А. считает необходимым сочетать импортируемые технологии и собственные научные разработки при решении вопроса технологического обновления производства [4]. Беляева А.О. говорит о необходимости повысить эффективность функционирования институтов фундаментальных и прикладных научных исследований [5].

В эру пятого технологического уклада важной проблемой для российской экономики являются возможности интернационализации, интеграции в европейское пространство, использования передового опыта высокотехнологичных стран, разработки федеральных и региональных инвестиционных программ, ориентированных на преодоление экономического отставания России от инновационноразвитых стран.

Использование имеющихся в достаточном количестве природных ресурсов совокупно с разработанным комплексом мероприятий по созданию стимулирующих условий для развития предпринимательской активности в инновационной сфере, позволит России эффективно использовать свой научно-технический потенциал и выйти на международный рынок с конкурентоспособной высокотехнологичной продукцией. 


\section{Выводы или заключение}

Проведённый нами анализ является лишь первым звеном комплексного исследования предпринимательской активности экономики России, которое предполагает изучение не только территориальных различий соответствующих индикаторов, но и измерение их взаимосвязи с другими явлениями, моделирование и прогнозирование. Статистика должна на практике стать инструментом обоснования научно-технической политики, оперативно реагируя на её приоритеты и не просто отражая сложившиеся тенденции, но и позволяя предвидеть их возможные изменения в будущем. Необходимо усилить внимание к проблемам выявления устойчивых закономерностей динамики научного и предпринимательского потенциала, прогнозирования количественных и структурных сдвигов. На основе обобщения мировых тенденций и опыта стран, находящихся на разных уровнях научно-технического развития, возможно сформировать концепцию динамики процессов развития науки и предпринимательства применительно к определенной макроэкономической ситуации, оценить действенность тех или иных вариантов научно-технической политики.

\section{Библиографический список}

1. Распоряжение Правительства РФ от 8 декабря 2011 г. №2227-р.

2. Михелашвили Н.Р. Перспективы развития российской модели национальной инновационной системы // Экономика и менеджмент инновационных технологий. - 2016. - № 1 [Электронный ресурс]. - URL: http://ekonomika.snauka.ru/2016/01/10696.

3. Удальцова Н.Л., Пиримова Л.С., Трубкина Я.Е. Современные проблемы национальной инновационной системы // Креативная экономика. - 2013. - № 6 (78). - С. 40-44.

4. Абрамова К.А., Джабарова А.Р. Актуальные проблемы внедрения инноваций в государственном управлении // Проблемы социально-экономического развития регионов: сборник статей Международной научно-практической конференции (8 мая 2015 г., г. Уфа). в 2 ч. Ч. 1. - Уфа: Аэтерна, 2015. -228 с. - C. 3-7.

5. Беляева А.О., Попова Е.О., Удальцова Н.Л. Барьеры, препятствующие развитию малого инновационного бизнеса в России // Экономические науки. - 2014. - №4(113). - С. 12-15. 